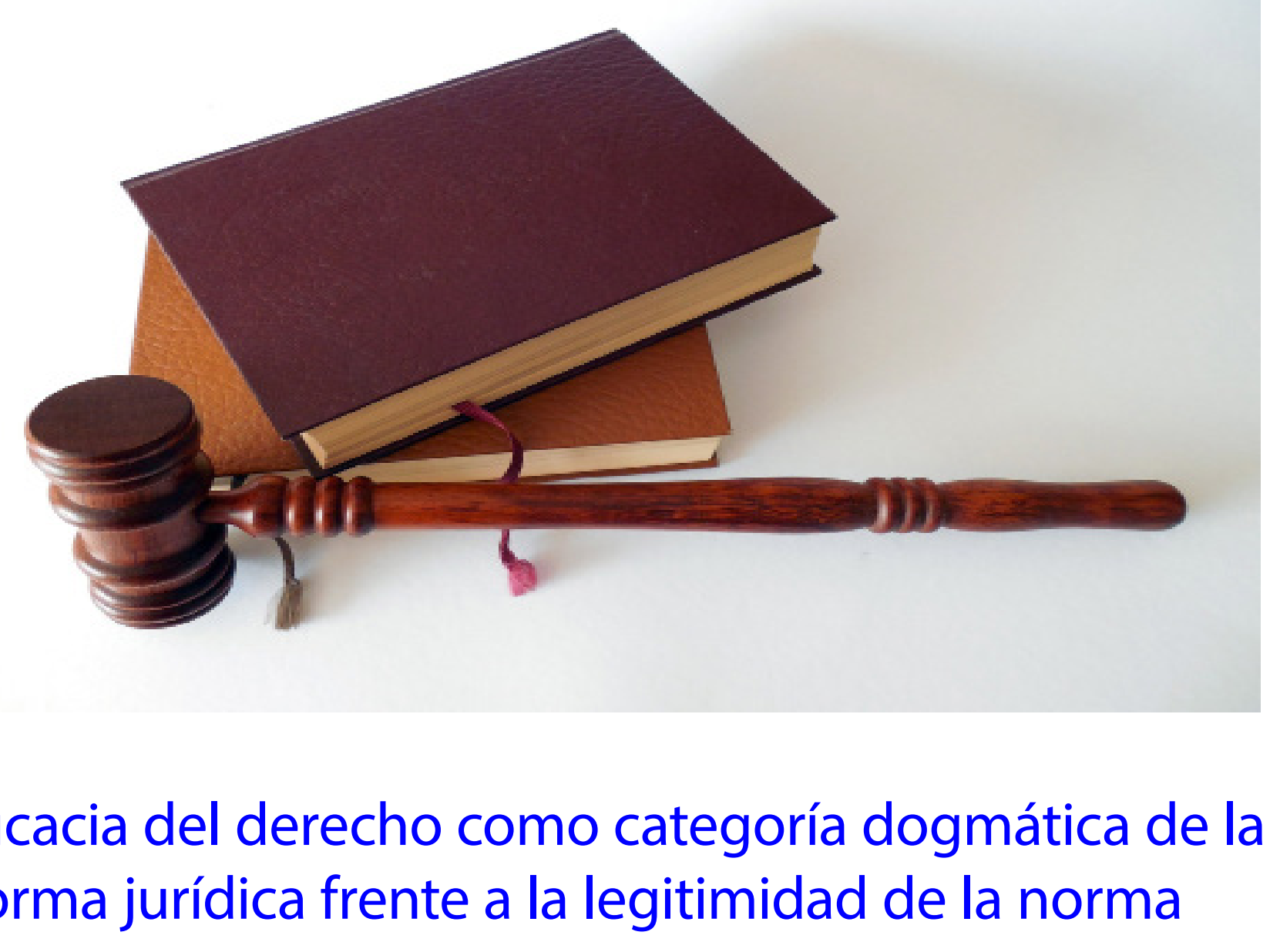

\title{
Eficacia del derecho como categoría dogmática de la norma jurídica frente a la legitimidad de la norma
}

\section{Effectiveness of law as a dogmatic category of the legal norm versus the legitimacy of the norm}

Indy Paola Nazir-Lleneris

Maestría en Ciencias Poliíicas, indy_1222@hotmail.com, https://orcid.org/0000-0001-9292-2886, Coordinadora de investigación de la Facultad de Ciencias Sociales de la Fundación Tecnológica Antonio de Arévalo - Unitectar, Cartagena de Indias, Colombia. 


\section{6}

Recibido: Enero 16 de 2019

Cómo citar: I.P Nazir-Lleneris, 'Eficacia del derecho como categoría dogmática de la norma jurídica frente a la legitimidad de la norma", Sostenibilidad, Tecnología y Humanismo, vol. 10, no. 2, 95-104, 2019.

Aceptado: Mayo 18 de 2019

Aceptado: Mayo 18 de 2019

(1)

(n)

(1)

(1)

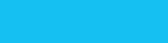

\section{Resumen}

El presente trabajo es producto de ciertas reflexiones teóricas, que involucran a la eficacia del derecho como categoría dogmática, frente a la legitimidad del ordenamiento jurídico colombiano. Con este trabajo se dan elementos al lector que le permiten establecer como la ineficacia, no es de recibo en las organizaciones del orden estatal, pero el sistema jurídico colombiano no está exento de la misma; en este sentido, se enuncia como nuestro sistema jurídico sufre un deterioro por falta de legitimidad por parte de los destinatarios directos de la norma. Así mismo permite mirar a Colombia desde una arista, que le concibe como un fracaso constitucional de las instituciones, que carecen de autoridad. Finaliza con una dura aseveración de como los colombianos no confían en sus instituciones y esto no favorece a la legitimidad de un derecho positivo, por tanto el ordenamiento se debilita, por falta de un verdadero capital social y una restructuración del orden legal.

Palabras clave: Eficacia, categoría dogmática, legitimidad. 
Introducción

Desde las sociedades más antiguas el incumplimiento normativo se mostró como un problema de orden social y jurídico; ya en las primeras civilizaciones se optó tanto por la coerción como por la coacción, para mantener el orden y preservar la paz, tratando de garantizar la reducción de los posibles momentos de crisis y desequilibrio [1].

En sociedades históricas como Babilonia, Grecia y Roma (máxima representación de las civilizaciones antiguas), se manejaba una disposición normativa con su respectiva sanción o consecuencia, encaminada a establecer el cumplimiento de dichas disposiciones; en Roma un caso particular era la Capitis Diminutio, que actualmente puede verse como la reducción de algunos de los atributos de la personalidad jurídica, o la pérdida de todos lo que la conforman, dependiendo del grado de ella es decir, mínima, media o máxima, está, entonces, era una de las tantas formas que los romanos utilizaron para sancionar e impartir justicia; vemos que desde los conceptos más remotos del derecho, la presencia del incumplimiento de este, creaba la necesidad y fundamentaba el hecho de que, cada norma ya fuera en si misma o en otra expresara una consecuencia o sanción coercitiva, constituyéndose como la parte dispositiva de la norma jurídica, que trataba de encaminar los comportamientos humanos y la conducta del ser hacia el acatamiento de la norma [2].

Esta estructura de la norma desarrollada en la antigüedad es la que hemos venido aplicando desde hace ya varios siglos, una parte hipotética y otra dispositiva; la que prevé y la que sanciona la conducta del ser; durante la elaboración del código civil francés, Napoleón se interesó por la costumbre de los ciudadanos de Francia, razonó sobre la importancia de sancionar y limitar algunos actos o hechos de los sujetos de derecho de tal territorio, partiendo de aquello, que se acostumbraba a hacer; en otras situaciones como por ejemplo el que hacer de la corona española durante la conquista, las disposiciones y las ordenanzas del rey, como máxima autoridad dotado de voluntad suprema, eran ley en España y en las nuevas tierras conquistadas, pero en estas últimas era casi imposible hacer valer las normas reales, de allí la famosa frase "el rey mandaba, pero no gobernaba", utilizada en muchos libros de historia para mostrar, como la voluntad del rey, siempre era frustrada por sus propios delegados [3].

Esta última, es quizás la herencia cultural y vivificada en el contexto histórico de América Latina, que aún sigue latente, por ello [4] en sus "notas preliminares para la caracterización del derecho en América Latina", nos menciona como el incumplimiento normativo que se traduce en ineficiencia del derecho, genera tantos problemas internos en el sistema normativo, al igual que en la implementación legal para la corrección y sanción, el hecho ya sea ilegal o ilícito; no sin dejar atrás la posición ambivalente de las políticas administrativas de los países latinoamericanos donde las decisiones legales están encadenadas, a las situaciones sociales, generando un dualismo particular, ante una misma controversia, por lo general se coloca por encima del derecho a los derechos individuales que este garantiza, provocando un fuerte sentimiento de independencia frente al derecho oficial, es como tratar de revelarse frente a lo que está establecido. Pero este no es el único problema, ya que median otros tales como el llamado "Trasplante Jurídico". 


\section{8}

Del trasplante jurídico y la ineficacia del Derecho.

Vale decir de inmediato, que el fenómeno de la ineficacia del Derecho, no es de recibo en las organizaciones del orden estatal, pero el ordenamiento jurídico colombiano no está exento del fenómeno en cuestión; vemos como nuestro sistema jurídico sufre un deterioro por falta de legitimidad por parte de los destinatarios directos de la norma, esto es una crisis social multifacética que el país atraviesa desde comienzos del siglo XVIII hasta nuestros días; mirar a Colombia desde este punto, es concebir un fracaso constitucional de las instituciones, que carecen de autoridad pues ellas mismas la han perdido, los colombianos no confían en sus instituciones y esto no favorece a la legitimidad de un derecho positivo, el ordenamiento se debilita ante nuestros ojos, por falta de un verdadero capital social y una restructuración del orden legal [5].

La historia colombiana nos ha demostrado, que la famosa fórmula de "copia y pega" conocido como trasplante jurídico, no resulta muy conveniente en la formación de nuevos Estados; después del arduo proceso de independencia y conceptos revolucionarios acerca de la libertad, la dignidad y la autodeterminación de los pueblos, el Estado en formación, no podía ser sometido a concepciones jurídicas provenientes del continente Europeo o aquellas disposiciones manejadas en Estados Unidos. Pero aun así, los gobernantes de la época intentaron disminuir los vacíos jurídicos presentes, en el nuevo Estado, introduciendo figuras de Francia y continuando con el régimen socio-jurídico que España nos impuso durante la conquista; fue uno de los más grandes errores de nuestros dirigentes políticos, intentar igualar las condiciones sociales de una Colombia en crisis, destruidas por las guerras exteriores y las civiles, que fueron secuelas del desorden y la mala administración del poder, con una realidad social Europea, en una situación de desarrollo eminentemente industrial, con una calidad de vida más estable que la nuestra.

Por ello, es prudente afirmar que Colombia avanza y retrocede al mismo tiempo, no hay una integridad en los procesos de desarrollo y mientras unos sectores despliegan otros siguen estancados o lo que es peor se desboronan poco a poco; no está demás decir, que las transformaciones sociales de los últimos tiempos, que en gran parte se deben a los avances tecnológicos, que han ido remplazando a otras máquinas y hasta al hombre, facilitando de cierta forma nuestro vivir, según muchos complicándolo más, no pueden dejarse pasar o echarse a un lado, es por esto que un "cambio social amerita un cambio normativo".

No acatar un comportamiento establecido por la ley o aquel que la misma sociedad permite, si no por el contrario, hacer lo que nos parece y decidir sin pensar en los efectos que estas decisiones generan, es un buen punto de partida e inspiración para miles de tratadistas que intenten establecer un porque o por lo menos algunas razones, que fundamenten este comportamiento o que conlleven a él; es por esto que más de uno ha dedicado una porción o gran parte de su tiempo, en realizar ciertas conjeturas en lo que a incumplimiento se refiere; autores como [6], han intentado establecer ciertas consideraciones; Nino, establece que una razón indiscutible es la falta de capital social, que en los últimos tiempos disminuye rápidamente, con esto se produce más desorden, conllevándonos a un desequilibrio democrático y a un estancamiento del desarrollo o lo que es peor a un desarrollo a medias, él formula una posible dosis para el anterior diagnóstico, y es que a partir de un comportamiento colectivo, de una culturización basada en el "nosotros" y no en el YO, y en una integración social como 
elemento dinamizador, se logre e impulse el progreso social.

Por otro lado aparece [5] en su libro "normas de papel", en donde pone de manifiesto su inconformismo con el manejo de la justicia en la sociedad Colombiana; afirma que aun cuando existan posibles factores de índole económico como la desigualdad, pobreza y la falta de apertura económica, esto no logra explicar el porqué de tanta criminalidad y violencia no solo en Colombia sino en toda América Latina, la falta de justicia ofrece grandes oportunidades para la expansión de la violencia a demás critica la tolerancia que es más que evidente cuando se trata de ilegalidad, por ello tanto individualismo e inmoralidad provoca un oleaje de corrupción casi imparable; según el citado autor, fomentar los valores post materiales como la autoexpresión y la mejor calidad de vida, traen como consecuencia un civismo que incide recíprocamente en el desarrollo, es por esto que para lograr un derecho que cumpla con los objetivos propuestos, al cual pueda atribuírsele el criterio de eficaz, se requieren sanciones más efectivas por parte del estado y una sociedad más activa que reproche ante el no acatamiento de la norma, solo así a partir de una convivencia ciudadana de la mano del poder público, se logrará una confianza mutua que conlleva a la cooperación y esto nos conduce a un bien colectivo que se traduce en bienestar y progreso.

Los aportes realizados [5], constituyen uno de los fundamentos más viables y apropiados en el momento de la práctica y la aplicación; las sociedades actuales pretenden que el estado a través de ciertas políticas solucione conflictos de orden social, no tratan de involucrarse en el proceso de resolución, son participes del conflicto directa o indirectamente porque lo generan o dejan que se genere y aun así dejan todo en manos de las instituciones estatales y no reconocen la importancia de su labor como promotores de soluciones efectivas, es necesario entonces, contar con una sociedad activa que no deje de lado sus responsabilidades como ente social, que se fundamente en principios éticos y morales y que no anteponga por encima de la ley a la costumbre o la religión, es necesario que la ley prevalezca ante ambas, sin que esto desmerite su importancia, un estado social armónico depende de ello; pero no solo depende, del conglomerado que lo conforma de su participación sino también del buen manejo de las funciones estatales y que las instituciones ofrezcan la mayor garantía para la aplicación eficaz de la justicia, esto legitima su poder, es decir los ciudadanos confían en sus instrucciones, respetan las leyes que promulgan y las cumplen, solo así el derecho logra ser eficaz.

Esto mismo ocurre con las instituciones colombianas, las cuales regulan y encaminan la realización de sus funciones a través de ciertas decisiones, relacionadas con el manejo de la justicia, la implementación de la legalidad, las facultades legislativas, las sanciones disciplinarias, las visiones reformistas y otras disposiciones estatales que están bajo su consideración, pero para tomar dichas decisiones, las instituciones se basan en unas razones, constituidos como esa facultad de discurrir, ese motivo, causa o argumento que indica la justificación de una acción o un fallo, que afecta indudablemente al Estado en su conducción de ente jurídico regulador de las relaciones de los sujetos entre si y de esta misma con el Estado; las instituciones Colombianas direccionadas por la condición del Estado social de derecho se encaminan al cumplimiento de las normas existentes en la constitución política, pero en ocasiones dejan de lado que la sociedad no puede ser vista como un espacio de dominación pues se encuentran excluidas de toda subestimación socio-política, el pueblo sede el poder pero siempre está bajo su guarda.

Una de las razones institucionales en el estado Colombiano, es aquella que cobija 


\section{0}

toda actuación por parte de los que dirigen la nación, es decir, el derecho simbólico que se va representando en nuestra carta política, su vigencia es a perpetuidad aun cuando al ser aplicada refleje su ineficacia, el simbolismo constitucional va más allá de su carácter supra legal, en realidad su valoración la obtiene de su misión básica: dirigir al estado Colombiano; durante su elaboración el pueblo le imprimió su carácter con una especial particularidad simbólica a partir de la aceptación de su contenido, donde de hecho se establece así misma como norma superior "norma de normas" (Art. $4^{\circ}$ de la C.N.) [1].

El simbolismo de la constitución se debe a que es el máximo fundamento del orden jurídico, que ha sido desarrollado por una estructura positiva especificada para cada Estado [7], plantea que no hay Estado sin constitución, por ello el simbolismo constitucional va de la mano de la supremacía de la constitución; la constitución como norma de normas y ley de leyes simboliza la unidad del ordenamiento, constituyéndose como el primer fundamento de la organización estatal, gracias a ella se establecen relaciones justas y equitativas entre el poder, los gobernantes, los gobernados y las instituciones estatales, cada relación con su respectiva regulación.

Dentro de un marco de derecho se trata de establecer, la verdadera jerarquía existente entre los diferentes niveles de la norma, ya [7] en su teoría para el derecho, proponía la teoría de la pirámide jurídica; en los análisis realizados a la estructura jerárquica del orden jurídico, Kelsen tratando de responder el porqué de la unidad del ordenamiento y donde reposa la validez de toda norma que lo conforma, supone la existencia de una norma fundamental y única, estableciendo que después de ella aparece la constitución, representando el nivel más alto dentro del derecho nacional, simbolizando la unidad de todo ordenamiento jurídico positivo y garantizando la validez de toda norma que lo compone.

Por otra parte el poder constituyente originario que está en manos del conglomerado, es el utilizado para la elaboración de la constitución, en los procesos de formación o reestructuración del estado, gracias a esto se manifiesta la voluntad soberana y se legitima la súper legalidad de la norma; el simbolismo constitucional también se deriva del contenido de la constitución, a partir de una parte orgánica se establece el sistema de gobierno, la forma de estado y se pone de manifiesto el régimen político, que se desarrolla más ampliamente en la parte dogmática, donde se reconocen los derechos de la persona y las garantías del ser, se limita la actividad del estado y se promulgan las libertades individuales, además de las normas neutras que le imprimen ese carácter rígido a las constituciones actuales; una constitución como norma fundamental contiene un sin número de normas que ponen de manifiesto y dejan entrever la filosofía política de un Estado, de allí la importancia de la constitución y la inexistencia del Estado sin esta disposición normativa, [8] define a la constitución como el sistema de fuentes formales del derecho, gracias a esto, a partir de la fuente formal fundamental, estableceremos el sistema jurídico del estado, además dice que es la primera de las normas de producción y la llama la "norma normarum". Como vemos la constitución se funda en una pretensión de permanencia y duración lo que también le imprime ese carácter superior ante cualquier norma ordinaria.

Autores como [9], manifestó que la unidad del ordenamiento jurídico que pretende ser pleno, se basa en la constitución tomada como primer fundamento positivo del orden y la razón de validez de toda norma que lo conforma o que pretende conformarlo, he ahí que la coherencia del ordenamiento depende de que toda norma 
ordinaria se fundamente y no contradiga a la norma superior; por lo anterior la supremacía de la constitución, sustenta el simbolismo de esta y este último se constituye como la máxima razón institucional de vigencia de la norma a pesar de su ineficacia.

Aún cuando la anterior razón institucional, fundamenta la vigencia de ciertas normas ineficaces, existen ciertos criterios para que una norma sea eficaz, de hecho importantes tratadistas y doctrinantes [9]-[10] en sus obras más relevantes, nos los enuncian como criterios de la norma jurídica que ha nuestro parecer son los cimientos para alcanzar una óptima aplicabilidad del derecho, por lo general se habla de justicia, validez y eficacia, casi siempre enunciados como problema deontológicos, ontológicos y fenomenológicos del derecho, pero en nuestro caso la justicia tiende a ser un tanto subjetiva y es por esto que se engloba en el concepto de legitimidad.

Cuandomencionamosqueunaregladederecho positivizada es válida nos fundamentamos en su armónico desarrollo en Pro de la norma fundante, su existencia y permanencia dentro del ordenamiento, es decir su vigencia, y por último que en el momento de ser promulgada la autoridad tuviera competencia para hacerlo, es básicamente esto lo que garantiza la validez de la norma y es el primer paso para su eficacia; a partir de este primer paso surge el reconocimiento por parte de los ciudadanos, cuando el conglomerado reconoce y considera a la norma justa la legítima, pero además de esto el grupo social que se rige por dicha disposición normativa, debe confiaren sus instituciones, creer que tienen la autoridad, la competencia y la moral suficiente para dirigir y dirigirse a sí mismos, para ser ejemplos de vida, modelos a seguir y máximos exponentes del justicia, esto es, cumplidores en potencia; he aquí el más grande inconveniente para la eficacia de la norma, en especial en América latina y particularmente en Colombia, donde nuestros dirigentes políticos no llenan casi ninguno de los requisitos mencionados $y$ esto desvirtúa la confiabilidad de nuestras instituciones, alegando entonces que resulta paradójico cumplir aquello que ni siquiera el que lo hace y elabora lo cumple [11].

Es posible afirmar que son estos los criterios fundamentales, algunos de ellos más evidentes que otros, pero todos partes integrantes de un todo normativo; además de los mencionados nos dimos la tarea de crear dos nuevos criterios que participan activamente en Pro de la eficacia, como son una acción por parte de la autoridad y una acción por parte de los ciudadanos, ya que es posible que la norma sea legitima y se manifieste eficazmente, pero resulta vago creer que por una validez, vigencia, justicia y legitimidad la norma sea eficaz, constante y permanente; cuando hablamos de una acción por parte de la autoridad hacemos referencia a un nuevo papel mucho más integro que debe ser asumido por los funcionarios públicos, las autoridades no solo deben garantizar la realización del supuesto y la respectiva sanción como consecuencia del incumplimiento, sino que además debe motivar al capital social, a partir de una promoción cultural al cumplimiento de la norma, y deben pretender crear nuevos cumplidores natos, con conciencia normativista, es fundamental que la justicia a través de los medios estipulados por la ley esté al alcance de todos, por otro lado la coerción es fundamental para el cumplimiento, si no hay coerción no hay garantía, y sin esta ultima las normas se convierten en simples recomendaciones, es por esto que se debe minimizar la flexibilidad, toda sanción debe ser justa y equitativa, es indispensable que la equidad prime y que la autoridad cumpla con lo que instituye y crea [12].

Al mencionar una acción por parte de los ciudadanos retomamos lo dicho a grandes rasgos que "el pueblo siempre tiene el poder y lo cede a sus dirigentes", pero ninguna sociedad 


\section{2}

se formará y llegará a un pleno desarrollo hasta que comprenda que un dirigente no cambia al mundo, regenera la historia, motiva las controversias y hasta se cambia así mismo, pero no logra cambiar una estructura social si la sociedad así no lo cree, sin participación activa de ella no es nada; el ciudadano debe concientizarse de su importancia social y de su deber como ente social, es más que necesaria su actuación y manifestación indeleble en busca de un sistema más justo y eficaz, por ello se exige menos tolerancia ante la ilegalidad y más reproche ante el incumplimiento, mas fomento a la cultura y a la integración social, así dejaremos el "yo" y pensaremos en el "nosotros", un capital social cívico que incida en recíprocamente en desarrollo, la convivencia armónica entre cultura, moral y ley donde esta última prima, una participación democrática activa y en rechazo a la impunidad y el fomento al cumplidor nato que haga caso omiso, al carácter relativo de la norma; a nuestro parecer estos dos últimos criterios enunciados como acciones, considerados por muchos como superfluos, pero en realidad son los últimos pasos para alcanzar una eficacia contante.

Hasta ahora es posible afirmar que el derecho es una herramienta de emancipación social y que intentar desarrollar al derecho en un contexto diferente al político y el social es imposible; la sociedad está conformada por unos actores sociales, unos comportamientos y pensamientos que se organizan y relacionan entre sí, y el derecho es la organización puesta en ejecución por medio de la justicia; el derecho puede darse como un mecanismo para cambiar el comportamiento social y si se ve de esta forma se convierte en un elemento revelador para comprender a la sociedad y caracterizar su producción dentro del fenómeno político, no resulta conveniente concebirlo como una referencia o instrumento de dominación, es mejor considerarlos como un recurso que puede ser utilizado para el desarrollo político de los dominados y una visión más prospera de los gobernantes [13].

Por otro lado es posible identificar otra gran razón institucional que en Colombia permiten la vigencia de la norma a pesar de su ineficacia, y esta es la llamada ineficacia en términos instrumentales, según [5], en ocasiones el problema de la eficacia radica en la técnica de la implementación legal que deja de lado hechos muchos más importantes, dicha técnica errada se origina en las misma $s$ instituciones, por ellos es frecuente que el derecho en América latina no sea creado para ser eficaz o por lo menos de manera prioritaria, es decir, no se considera indispensable y fundamental, por ello se ratifica la existencia de una difundida practica institucional que consiste en crear normas para satisfacer necesidades institucionales de legitimidad más que para lograr propósitos instrumentales, esto ocurre por la marcada crisis moral, que pasan en este momentos nuestras instituciones, la sociedad no reconoce su competencia y las tilda de "corruptas", por ello en ocasiones las normas jurídicas son ineficaces en términos instrumentales, dado que apuntan a cumplir propósitos distintos a aquellos que se desprenden de la lectura espontánea de sus textos, en Colombia esto es muy frecuente, en especial en el código civil, que por su antigüedad y redacción clásica tiende a ocultar un sinfín de disposiciones que resultaría casi imposible determinar, sin antes realizar una investigación e indagación integra en todos sus artículos; la norma pretende ser completa pero termina siendo abstracta y oscura, esto facilita su ineficacia.

\section{Resultados}

Con esta investigación se pretende, demostrar cómo cada cosa depende de otra el derecho es un gran reloj indestructible que jamás se detiene, es un ciclo continuo, a veces correcto $y$ en otras ocasiones no tanto; todo reloj 
está formado por piezas y una sola pieza no garantiza que este se mueva, y lo mismo ocurre si una sola pieza falta, es por esto que a lo largo de la investigación hemos realizado un entrelazamiento de todas y cada una de las piezas, motivos razones, criterios o como gusten llamarlos para ratificar de esta forma que el derecho se mueve en la sociedad como esta así lo desee, es probable que se estanque por un comportamiento negativo ya sea social o institucional, pero ante todo por crisis que no se prevén o aquellas que no previstas no se logran resolver o por lo menos controlar.

Recapitulandotodos estas posiciones podemos concluir que es posible la existencia de una norma valida, pero que al no ser legitima tiende a ser ineficaz, es probable que la ineficacia se sustente en su mala elaboración, es decir, leyes que por su contenido defectuoso tienden a ser inaplicables a la realidad actual o a aquellas que por su existencia de antaño han caído en desuso, pero en otras ocasiones la norma no está mal diseñada cumple con los requisitos y no está fuera del contexto social, entonces surge el interrogante ¿Por qué la norma es ineficaz? La respuesta que hemos estado buscando nos muestra que aun con todo lo mencionado la norma no logra ser legitima; por ello la relación es más que evidente entre validez, eficacia y legitimidad que pueden ser considerados como los peldaños de la escalera a un orden jurídico más justo y eficiente, que acorte la brecha entre el derecho y la realidad. A nuestro parecer "el derecho vigente solo será eficaz cuando sea legítimo" [14], [15], [16].

\section{Referencia}

[1] Constitución política de Colombia. Título I y II. Bogotá (Colombia): Leyer, 2019

[2] Código Civil Colombiano. Título preliminar. Bogotá (Colombia): Leyer, 2009

[3] Ley 708 de 2002. Regímenes de caños, lagunas interiores y playas. Congreso Colombiano.

[4] M. Villegas, Normas de papel. Colombia, 2009

[5] J. Rodríguez, "Crisis en la legitimidad del Estado, del derecho y de la democracia liberal". Pensamiento Jurídico, no. 41, pp. 105-126, 2015

[6] C.S Nino, Un país al margen de la ley. Buenos Aires: Ariel, 2005

[7] V. Naranjo, Teoría constitucional $\boldsymbol{e}$ instituciones políticas, Colombia. Unión, 2008

[8] R. Álvarez, "Las fuentes de conocimiento de lo jurídico", Boletín Mexicano de Derecho Comparado, vol. 47, no. 139, pp. 39-63, 2014

[9] J.Ruiz, "Bobbioy los conceptos denormajurídica últimamente", Secuencia (Florianópolis), vol. 64, pp. 39-56, 2012

[10] E. García, Introducción al estudio del derecho, Primera edición en 1940, 2002

[11] H. Kelsen, Teoría pura del derecho. Bogotá (Colombia): Unión, 2000

[12] P. Navarro, Ensayo. Eficacia, tiempo y cumplimiento, 2009

[13] Y. Sanz, Visión panorámica de las causas de ineficacia del acto jurídico Testamentario; especial referencia a la revocación. Universidad de las Tunas, 2009

[14] M.J Gonzales, Ineficacia, anomia y fuentes del derecho, España - Madrid. Dikinson, 2003

[15] S.Y Garcia Salazar, J.F. Rojas-Ovalle y E.J. Barrientos Monsalve, "Alcance de las responsabilidades de las fiscalías de Venezuela y Colombia en la franja fronteriza "estudio de caso por homicidios", Mundo Fesc, vol. 8, no 16, pp. 78-85, ene 2019 
Eficacia del derecho como categoría dogmática de la norma jurídica frente a la legitimidad de la norma

\section{4}

[16] M. Prieto, Ensayo. Validez, vigencia, eficacia

y legitimidad, relación y distinción, 2001 